Vol. 17, n 1 | 2013

Varia

\title{
La relégation des femmes récidivistes en Guyane française (1887-1907)
}

Jean-Lucien Sanchez

\section{(2) OpenEdition \\ Journals}

Édition électronique

URL : https://journals.openedition.org/chs/1413

DOI : $10.4000 /$ chs. 1413

ISSN : 1663-4837

Éditeur

Librairie Droz

Édition imprimée

Date de publication : 1 mai 2013

Pagination : 77-100

ISBN : 978-2-600-01718-2

ISSN : 1422-0857

Référence électronique

Jean-Lucien Sanchez, "La relégation des femmes récidivistes en Guyane française (1887-1907) »,

Crime, Histoire \& Sociétés / Crime, History \& Societies [En ligne], Vol. 17, $n^{\circ} 1$ | 2013, mis en ligne le 01 mai

2016, consulté le 22 mars 2022. URL : http://journals.openedition.org/chs/1413 ; DOl : https://doi.org/ $10.4000 /$ chs. 1413

Ce document a été généré automatiquement le 22 mars 2022.

(c) Droz 


\title{
La relégation des femmes récidivistes en Guyane française (1887-1907)
}

\author{
Jean-Lucien Sanchez
}

1 Durant près d'un siècle, de 1852 à 1953, la Guyane française a été synonyme de terre de bagne et a reçu un peu plus de 70000 forçats. La loi du 30 mai 1854 sur l'exécution de la peine des travaux forcés, dite loi sur la transportation, répondait à un triple objectif : débarrasser définitivement le sol de la métropole de certains criminels; fournir à la colonie une main-d'œuvre abondante et bon marché pour assurer son développement ; et permettre aux plus méritants des forçats de s'installer en concession ou de bénéficier d'un engagement de travail à leur sortie du bagne afin de favoriser leur reclassement. Pour répondre à ce dernier objectif, le législateur offrit la possibilité à des femmes condamnées aux travaux forcés de choisir le chemin de l'exil en Guyane ou bien de purger leur peine dans des maisons centrales. La loi sur la transportation prévoyait ainsi dans son article 4 que «Les femmes condamnées aux travaux forcés pourront être conduites dans un des établissements créés aux colonies [...]». Cette mesure visait essentiellement à permettre l'union de forçats afin de favoriser leur installation durable sur le sol de la colonie. Près de 394 transportées ainsi que 7 déportées et 60 à 70 réclusionnaires furent donc envoyées en Guyane à partir de 1853 jusqu'en 1881². L'arrivée des premières transportées à Saint-Laurent-du-Maroni, le principal point d'accueil des transportés à partir de 1857, débute en 1859. Là, elles étaient confiées à la garde des sœurs de l'œuvre de Saint-Joseph de Cluny et maintenues dans un dépôt surnommé le couvent.

2 L'objectif du législateur de la transportation était fortement influencé par l'expérience conduite en Australie par la Grande-Bretagne. L'enjeu étant de permettre l'éclosion d'une colonie de peuplement susceptible de concourir à l'enrichissement de l'empire colonial français. Mais les conditions de vie sur place sont dramatiques et le taux de mortalité particulièrement élevé : sur près de 21620 transportés expédiés en Guyane à partir de 1852, il n'en restait plus que 7466 en $1866^{3}$. Face à cet échec, le gouvernement 
recule et décide à partir de 1871 d'envoyer les transportés européens en direction de la Nouvelle-Calédonie, au climat beaucoup plus clément, et de ne réserver la Guyane qu'aux transportés coloniaux. Malgré ce précédent, le législateur décide à partir de 1887 d'envoyer à nouveau des femmes condamnées en Guyane. Plus précisément des reléguées, frappées par la loi sur la relégation du 27 mai 1885. Mais à l'inverse de leurs homologues transportées, ces femmes n'ont pas eu le choix de décider de leur sort et devaient obligatoirement purger leur peine dans la colonie.

3 L'historiographie des bagnes de Guyane est relativement bien connue notamment grâce aux travaux pionniers de Michel Pierre. Publié en 1982, La terre de la grande punition a ouvert la voie à de nombreuses recherches sur cette thématique. À sa suite, Danielle Donet-Vincent, à travers deux ouvrages majeurs ${ }^{4}$, a effectué un travail de fond considérable en exploitant des sources totalement inédites. Elle a notamment mobilisé les archives des Jésuites qui assistèrent à l'installation des premiers transportés en Guyane ainsi que celles de deux acteurs majeurs dans le processus d'abolition du bagne que furent le capitaine de l'Armée du Salut, Charles Péan et le député de la Guyane, Gaston Monnerville. Ces sources sont incontournables pour saisir l'histoire du bagne et l'ouvrage de De soleil et de silences. Histoire des bagnes de Guyane, demeure à ce jour l'ouvrage de référence en la matière. La colonisation pénale de la Guyane a également connu un important investissement dans le monde anglo-saxon ${ }^{5}$. En ce qui concerne plus précisément la question des femmes condamnées au bagne, Odile Krakovitch leur a consacré un important ouvrage intitulé Les femmes bagnardes. Il a pour particularité de traiter de toutes les catégories de condamnées (transportées, relégués, déportées et réclusionnaires) et brosse une étude particulièrement complète puisqu'elle comprend tout à la fois le cas du bagne calédonien et celui de la Guyane.

4 L'objet du présent article s'inscrit dans la continuité des travaux d'Odile Krakovitch et se propose, sur la base d'archives inédites, d'analyser exclusivement le sort des femmes reléguées en Guyane. La principale source disponible sur ce thème se situe au sein de la série Colonies $\mathrm{H}$ aux Archives Nationales d'Outre Mer. Ce fonds correspond aux archives de l'administration pénitentiaire en Guyane. Mais une autre source relativement peu exploitée est également disponible aux Archives départementales de Guyane, au sein de la série IX Administration Pénitentiaire. Il s'agit des archives du gouverneur de la colonie qui avait, à travers l'action du directeur de l'administration pénitentiaire placé sous sa " haute autorité », la charge de la direction du bagne. Les archives des sœurs de l'œuvre de Saint-Joseph de Cluny donnent quant elles de précieuses indications sur le quotidien des reléguées. Bien qu'utilisées par Odile Krakovitch, nous avons pu au cours d'un séjour de recherche exploiter l'intégralité de ces archives qui ont bénéficié dans l'intervalle d'un classement complet.

Comme pour les transportées, les reléguées sont expédiées en Guyane essentiellement pour servir d'épouses aux relégués et permettre leur installation durable sur le sol de la colonie. Mais ces femmes vont subir une peine que le législateur métropolitain n'avait pas prévue et essuyer un sort particulièrement tragique. Placées sous la tutelle de leurs maris ou sous celle des sœurs de l'œuvre de Saint-Joseph de Cluny, elles demeurent des mineures qui ne peuvent pas disposer d'elles-mêmes. Ainsi, en quoi l'application de la relégation des femmes en Guyane excède-t-elle largement le cadre fixé par la loi ? Dans quelle proportion la différence des sexes structure la dissymétrie de traitement qui leur est réservé par rapport à leurs homologues masculins? Et de quelles alternatives 
disposent-elles pour s'émanciper de la condition dans laquelle les réduit l'administration pénitentiaire?

6 Pour répondre à ces différentes questions, il faut en premier lieu questionner l'objet même de la loi sur la relégation et saisir en quoi cette mesure constitue en elle-même, pour les hommes et plus encore pour les femmes qui en sont frappés, un régime largement exorbitant du droit commun. L'analyse du régime collectif des reléguées collectives et de leurs conditions de vie au sein du couvent de Saint-Laurent permet ainsi d'apporter un premier élément de réponse. Il est nécessaire ensuite de s'étendre en second lieu sur les stratégies déployées par les reléguées pour échapper au régime du bagne et sur les opportunités dont elles disposent pour s'en affranchir. Ce faisant, il demeure en dernier lieu à analyser l'échec de cette mesure symbolisé par le long processus d'abolition de la relégation des femmes en Guyane.

\section{La relégation des récidivistes : une mesure exorbitante du droit commun}

7 La loi du 27 mai 1885 sur la relégation des récidivistes entraîne l'internement à perpétuité sur le sol d'une colonie de délinquants et de criminels récidivistes. Cette mesure aménage un quantum, c'est-à-dire plusieurs combinaisons de peines qui, si elles sont toutes inscrites au casier judiciaire d'un récidiviste, sont censées démontrer matériellement son caractère incorrigible et entraînent le prononcé obligatoire pour le magistrat de la peine de la relégation ${ }^{6}$.

8 Toutefois, si elle élimine socialement du sol de la France métropolitaine et de certaines colonies des condamnés récidivistes, la relégation aménage également un régime destiné à leur permettre de s'installer sur le sol de la colonie afin d'y faire souche. Mais ce régime repose sur une distinction censitaire qui entraîne un dévoiement total de l'application de la relégation en Guyane. Les relégués les mieux notés et qui disposent de moyens financiers suffisants pour se prendre en charge sont classés au régime de la relégation individuelle. Ils bénéficient d'un régime de liberté et ont la possibilité d'obtenir un engagement de travail ou de s'installer en concession. Mais ceux qui ne disposent pas de moyens financiers suffisants, c'est-à-dire l'immense majorité d'entre eux, sont pris en charge par l'État et doivent donc travailler pour le compte de l'État. Cette contrepartie se traduit dans les faits par un internement au sein d'un pénitencier, celui de Saint-Jean-du-Maroni, où les relégués collectifs sont soumis à des travaux forcés. Dans l'esprit du législateur, le régime de la relégation collective est provisoire. Il est destiné à préparer le relégué aux travaux coloniaux et à lui permettre d'accumuler un pécule suffisant pour pouvoir à terme s'installer en tant que colon, c'est-à-dire de bénéficier du régime de la relégation individuelle.

9 Le régime des relégués collectifs en Guyane est donc en tout point comparable à celui des transportés, qui sont internés eux au sein d'un pénitencier situé à Saint-Laurent. La relégation représente donc une atteinte considérable au principe de proportionnalité de la peine au délit. Car $80 \%$ des condamnations à la relégation en Guyane sont prononcées par des tribunaux correctionnels contre des délinquants coupables de vol simple, de vagabondage et de rupture de ban. Les relégués doivent donc purger dans un premier temps leur peine d'emprisonnement. Puis ils sont soumis dans un deuxième 
temps à une mesure de sûreté qui les condamne en Guyane à une peine de travaux forcés, peine réservée d'ordinaire à des criminels condamnés par des cours d'assises.

En outre, à l'inverse de la transportation, la relégation s'applique obligatoirement aux femmes. Par " récidivistes ", la loi entend toutes les personnes ayant récidivé et ce quel que soit leur sexe. Près de 519 femmes, coupables essentiellement de vol simple et condamnées à plus de $96 \%$ par des tribunaux correctionnels, rejoignent ainsi la Guyane de 1887 à 1904. Comme pour les hommes, elles peuvent être placées soit en relégation collective, soit en relégation individuelle. Celles placées en relégation individuelle peuvent sur leur demande ou d'office si des «moyens honorables d'existence " leur font défaut, être placées dans des maisons d'assistance et de travail où « il est pourvu à leurs besoins ». Le décret du 26 novembre 1885 portant règlement d'administration publique pour l'application de la loi du 27 mai 1885 sur la relégation des récidivistes ajoute que les reléguées individuelles peuvent y être maintenues jusqu'à ce qu'elles aient trouvé un engagement de travail ou «à s'établir dans des conditions suffisantes de bon ordre et de moralité ». C'est-à-dire jusqu'à ce qu'un mari les réclame.

Le régime des femmes placées en relégation collective est quasiment identique si ce n'est que pour obtenir tous les avantages accordés aux reléguées individuelles, les reléguées collectives doivent simplement justifier « d'une bonne conduite et d'aptitude suffisante ». Mais le manque de précision de ce décret et le peu d'intérêt témoigné par les autorités pénitentiaires de la colonie pour ces femmes entraînent une application de la loi en Guyane très éloignée des objectifs fixés par le législateur métropolitain.

\section{L'installation des reléguées à Saint-Laurent}

À Saint-Laurent, les sœurs de l'œuvre de Saint-Joseph de Cluny, présentes en Guyane depuis 1822, s'occupent de la surveillance du dépôt des transportées et assurent l'enseignement des enfants des deux écoles du village (filles et garçons) ${ }^{7}$. L'administration pénitentiaire recourt effectivement massivement aux congrégations religieuses féminines à partir de 1840 pour assurer la surveillance des femmes condamnées ${ }^{8}$. À partir du mois de juin 1887, les sœurs reçoivent également la mission de s'occuper des reléguées. Ainsi, à l'inverse des relégués qui sont encadrés par des surveillants militaires, les femmes sont placées sous la garde de religieuses, ce qui va grandement modifier leurs conditions d'incarcération. Le 24 juin 1887, 300 hommes et 24 femmes accompagnées par trois sœurs débarquent à Saint-Jean. Mais le camp destiné à leur internement n'étant pas prêt, ces femmes sont installées dans l'urgence au sein de l'école des garçons de Saint-Laurent :

Après une heureuse traversée de vingt jours, le navire mouillait à Saint-Jean, où l'on fit débarquer les 300 hommes relégués. Mais quelle cruelle déception éprouvèrent ces pauvres gens à leur arrivée dans cette petite île! On leur avait promis monts et merveilles, et ils n'aperçurent devant eux, d'un côté que le grand fleuve Maroni, et de l'autre d'immenses forêts impénétrables et marécageuses. Quelques arpents de terre seulement avaient été déboisés, et l'on y avait installé deux hangars, n'ayant pour toute couverture que des feuilles de cocotiers. C'est làdessous que se logèrent les 300 récidivistes ainsi que les gendarmes et les surveillants. Ceux-ci, à leur tour, furent étrangement et fort désagréablement surpris de ne trouver pour leur repas du soir que cinq ou six pommes de terre et quelques oignons laissés à leur disposition. Nous-mêmes et nos femmes, nous dûmes passer la nuit sur le bateau et redescendre le lendemain à Saint-Laurent [... $]^{9}$. 
13 L'installation des reléguées est ainsi particulièrement dramatique dans les premiers temps de leur arrivée car rien n'est prêt pour les accueillir en Guyane. Les convois se succèdent et portent leur nombre à 71 sous la garde des sœurs qui sont complètement dépassées par leur charge. La sœur supérieure estime ainsi qu'il faut affecter au minimum une sœur pour dix reléguées car « ces femmes sont beaucoup plus difficiles à surveiller que les condamnées ${ }^{10} »$. En janvier 1889 , le ministère des Colonies délègue trois religieuses supplémentaires pour assurer un service qui s'effectue au sein d'un dépôt totalement engorgé. À l'intérieur, les femmes (reléguées et transportées) ainsi que les enfants sont installés dans différents bâtiments, ce qui ne facilite pas la tâche de surveillance des sœurs :

Il y a près de 45 femmes qui logent à la maison attitrée autrefois à l'école des garçons, près de la rivière, que l'on appelle aujourd'hui la Relégation proprement dite ; celles-ci sont sous la surveillance de Mère [...], des sœurs Sainte Hélène et Suitbert. Le reste, sur 112 femmes, sont logées à la Transportation : une partie occupe le dortoir des filles ; l'autre partie couche à côté des femmes transportées ; les petits garçons dorment dans une chambre adjacente au dortoir des femmes transportées; les petites filles occupent pour la nuit la pièce appelée autrefois parloir ou le bureau de la mère supérieure. Heureusement que la cour est séparée par une palissade qui séquestre entièrement les femmes condamnées des femmes récidivistes; les garçons des filles. Sans cette palissade, la surveillance deviendrait de toute impossibilité. Déjà les petites filles, entendant les affreux propos des récidivistes, n'ont pas à gagner dans une pareille société dont elles sont proches voisines. Les sœurs sont obligées à être spectatrices du mal sans pouvoir y mettre fin, ainsi elles ne peuvent forcer les femmes ni à faire leur prière du matin et $d u$ soir, ni à assister à la sainte messe le dimanche, ni intercepter les lettres les plus horribles, les plus passionnées que les femmes adressent aux hommes récidivistes de Saint-Jean, ni les contraindre à une vraie discipline. [...] Elles assistent à des disputes continuelles qui s'élèvent entre ces femmes qui ne peuvent se supporter les unes les autres et où elles se reprochent mutuellement les choses les plus révoltantes $[. . .]^{11}$.

Ce n'est qu'à la fin de l'année 1889 que cette promiscuité prend fin et que les transportées sont déplacées dans un autre bâtiment. Après leur départ, le dépôt accueille exclusivement les reléguées ainsi que les petits garçons scolarisés par les sœurs. Les petites filles sont quant à elles scolarisées au sein du bâtiment réservé aux transportées. Toutefois, la présence des reléguées à Saint-Laurent est provisoire et n'est qu'une alternative en attendant l'achèvement des cases destinées à les recevoir à Saint-Jean. En mai 1889, ces cases sont enfin édifiées et l'administration pénitentiaire projette d'y envoyer les femmes l'année suivante. Néanmoins, lorsqu'elle se rend en inspection à Saint-Jean, la sœur supérieure se rend compte que ces installations sont insuffisantes et qu'elles ne peuvent accueillir la totalité de l'effectif dans des conditions décentes. De son côté, le directeur de l'administration pénitentiaire, qui ne parvient déjà pas à installer convenablement tous les relégués à Saint-Jean, est particulièrement embarrassé de devoir y accueillir ces femmes.

La sœur supérieure craint en outre que le déménagement des reléguées ne nuise à leur " moralité12 ». Consulté sur le régime disciplinaire prévu par le commandant du camp, ce dernier affirme à la sœur supérieure ne pas vouloir agir dans un but "moralisateur » mais n'envisager uniquement que l'aspect nuptial de cette installation. Pour ce dernier, les reléguées doivent être laissées en liberté et libres de contracter des mariages selon leur volonté. Dans l'optique de l'administration pénitentiaire, le rôle de ces femmes se résume à celui de futures mères destinées à assurer l'implantation durable des relégués 
au Maroni. Les autorités ont en effet encore à cœur de fonder à cette date un village de colons à Saint-Jean, et non un pénitencier comme il le deviendra rapidement par la suite. Outrée, la sœur supérieure menace d'abandonner l'œuvre et assure au commandant que les sœurs ne s'installeront jamais à Saint-Jean. Cette dernière se garde bien de partager l'angélisme des autorités pénitentiaires car les quelques tentatives de placement de reléguées à l'extérieur du dépôt de Saint-Laurent se sont toutes soldées par des échecs. Après une quinzaine de jours de liberté en moyenne, toutes les femmes sont réintégrées au dépôt pour des motifs de vol ou d'ivresse. La mère supérieure milite ainsi pour la pérennisation du dépôt de Saint-Laurent et pour l'application d'une discipline inflexible :

Ces femmes sont incorrigibles, il n'y a pas à compter sur elles ; on ne réussira jamais à leur faire du bien si elles ne doivent pas rester au dépôt, ce va et vient perpétuel occasionne de grands désordres sans aucun résultat. Il faudrait une discipline sévère pour ces femmes et que les sœurs eussent la latitude d'agir sans avoir besoin de recourir constamment à $\mathrm{M}$. le Commandant; qu'elles aient du travail pour les occuper, car elles remarquent bien que lorsque l'aiguille est maniée, il y a plus de silence, moins de chicanes $[. . .]^{13}$.

16 C'est désormais le visage que va prendre la relégation des femmes en Guyane. La sœur supérieure souhaite maintenir les reléguées à Saint-Laurent afin de les placer «à une grande distance des hommes pour empêcher toute relation ${ }^{14}$ » et le signifie à sa mère supérieure qui le signifie à son tour au ministre des Colonies. Contrariant le souhait initial du commandant de Saint-Jean, les sœurs s'occupent désormais à l'écart de la tutelle de ce dernier de «leurs femmes » et décident du régime le mieux approprié pour elles, c'est-à-dire un régime monacal particulièrement austère. Le dépôt des reléguées est d'ailleurs surnommé le couvent par les habitants de Saint-Laurent et par les reléguées elles-mêmes.

\section{Le couvent des reléguées et les différentes missions des sœurs}

17 En 1890, le ministre des Colonies ordonne donc de maintenir définitivement les 175 reléguées présentes en Guyane à Saint-Laurent. Cette situation est toutefois totalement contraire à la loi sur la relégation des récidivistes qui précise que tous les relégués, femmes incluses, doivent résider sur un territoire distinct de celui des transportés ${ }^{15}$. Mais cet aménagement n'est que la première entorse faite au régime de ces femmes dans la colonie.

18 Le couvent organisé par un arrêté en date du 25 septembre 1895 est composé de deux bâtiments. Le premier est réservé aux sœurs réunies au sein de la communauté de Sainte-Madeleine au Maroni (Relégation). Les sœurs sont installées à l'origine dans un dortoir en « piteux état » dans lequel elles sont obligées d'ouvrir « des parapluies » les jours de pluie. En octobre 1893, le gouverneur leur accorde un nouveau logement laissé libre par des religieux qui se situe à peu de distance du dortoir des reléguées. Il leur permet d'avoir un lieu qu'elles ne partagent plus avec les condamnées et dans lequel elles peuvent recréer un semblant de communauté. Le second bâtiment du dépôt comprend trois dortoirs pouvant recevoir chacun 63 pensionnaires. Ces dortoirs sont construits dans des bâtiments en rez-de-chaussée où les parois sont formées de tôles galvanisées et sont donc "inhabitables » en journée du fait de la chaleur «torride » 
qu'il y règne. Un de ces dortoirs reçoit également les lits des enfants des reléguées car les mères ne sont pas séparées de leurs enfants durant leur internement.

L'arrêté du gouverneur organisant ce dépôt prévoit que sa direction soit confiée à un chef de dépôt assisté par des sœurs "auxiliaires». Ainsi, c'est au commandant supérieur de la relégation qu'incombe normalement la charge de le diriger. Dans les faits, c'est le commandant du dépôt de Saint-Laurent qui y exerce toute autorité et qui en délègue une grande partie à la sœur supérieure. Cette dernière doit veiller à sa bonne tenue et assurer la discipline au sein des reléguées mais également au sein des sœurs de la communauté. Il arrive ainsi assez fréquemment qu'elle réclame auprès de sa mère principale leur remplacement car certaines d'entre elles peuvent se montrer réfractaires. Toutes ne s'acclimatent pas facilement de leur mission et souffrent des réactions ou des paroles des pensionnaires dont elles doivent assurer la garde.

Les sœurs attachent également une attention très importante à la direction spirituelle et morale des reléguées. Ces femmes, relativement âgées pour la plupart, observent en règle générale leurs devoirs chrétiens. Presque toutes font partie de l'apostolat de la prière et un bon nombre d'entre elles effectuent leur communion mensuelle. Car tous les dimanches et jours de fêtes, la messe est dite par un père de la Congrégation du Saint-Esprit à la chapelle du dépôt. Et lors des principales fêtes religieuses, ce sont les reléguées elles-mêmes qui s'occupent du chant et s'en acquittent avec «beaucoup de soin et de gravité ». Les sœurs parviennent même à convertir certaines d'entre elles. En 1890, deux reléguées protestantes acceptent ainsi de se convertir au catholicisme et abjurent « leurs erreurs » devant leurs compagnes. De nombreux cas de conversions et de baptêmes de reléguées sont répertoriés par les sœurs. La foi et le recours à la religion semblent effectivement un viatique un peu revigorant pour ces femmes au sein d'une existence où il ne leur reste guère d'autre réconfort.

21 Les sœurs sont également chargées de l'enseignement au sein des deux écoles de SaintLaurent. À cet effet, le dépôt comprend une étude où les élèves peuvent faire leurs devoirs après la classe. La rentrée scolaire a lieu d'ordinaire au mois d'octobre et les sœurs accueillent en moyenne 75 à 80 enfants. Sur ce nombre, une trentaine sont internes et sont pour la plupart orphelins. Ils sont pupilles d'État et logés dans un internat régi par les sœurs. À partir de treize ans, les garçons sont normalement placés tandis que les filles restent aux côtés des sœurs qui leur apprennent un métier manuel jusqu'à ce qu'elles soient en âge de se marier. Les externes sont essentiellement des enfants de concessionnaires pénaux et des enfants de fonctionnaires locaux. Les enfants de condamnés et ceux du personnel libre sont donc mélangés au sein des écoles. Mais cette promiscuité gêne le personnel administratif ainsi que les différents visiteurs officiels qui se succèdent au couvent. Le procureur de la République s'étonne ainsi en 1896 de voir le fils du juge de paix de Saint-Laurent partager les mêmes bancs que les enfants des concessionnaires pénaux ou des femmes condamnées. Quant aux enfants des reléguées qui vivent à leurs côtés au sein du dépôt, l'inspecteur des colonies Picquié réclame en 1896 leur isolement dans une ferme-école :

Je me trouve en face du grand dortoir des femmes dans l'enceinte même du pénitencier, où on a placé par un singulier oubli du respect dû à l'enfance, le dortoir des enfants d'origine pénale. On devine quels spectacles et quels exemples ces malheureux ont journellement sous les yeux, quel trouble et quelle excitation malsaine doivent apporter à ces pubertés en éveil, l'obscénité de langage et d'attitude de cent drôlesses éhontées. Cet exemple les poursuit jusque dans leurs études. C'est devant leur classe que les condamnés en quête de femme viennent 
faire leur choix; sous leur regard que s'ébauchent ces unions singulières, en des termes et avec des gestes qui ne constituent pas précisément un enseignement ${ }^{16}$.

À partir de 1900, des parents d'origine libre se plaignent à leur tour du mélange opéré entre leurs enfants et celui des condamnés au sein des deux écoles :

Je vous suis bien reconnaissante ma bien chère Mère, de m'avoir renvoyé ma sœur Ramolet; aucune des trois sœurs qui l'ont remplacée pendant son congé n'ont voulu se faire à cet emploi [d'institutrice], le trouvant trop assujettissant et ingrat. Cela est vrai, il faut à ces pauvres enfants, dépourvus pour ainsi dire de toute bonne qualité, des maîtresses dévouées, désintéressées, travaillant uniquement pour Dieu et le bien de ces jeunes infortunés. Je parle ici des enfants de condamnés, ceux du personnel libre sont un peu plus satisfaisants, mais leurs parents souffrent des contacts que ces derniers ont avec les premiers, et ils désirent vivement une classe à part. Cette question a provoqué la construction d'un bâtiment que les uns appellent déjà l'école laïque ! Nous ne savons pas ce que le bon Dieu nous réserve à ce sujet ${ }^{17}$.

laintes finissent par porter leurs fruits et en février 1904, le sous-directeur de l'administration pénitentiaire décide de procéder à la séparation au sein des deux écoles des enfants d'origine libre avec ceux d'origine pénale. Les enfants d'origine libre sont désormais confiés à une institutrice et à un instituteur laïques et sont placés dans une nouvelle école construite spécialement pour eux. Puis au mois de mars 1906, l'instruction des enfants d'origine pénale est également retirée aux sœurs pour être également confiée au personnel laïque. À compter de cette date, la mission des sœurs se limite désormais à la surveillance des reléguées et transportées installées au couvent.

\section{L'organisation du travail au sein du couvent}

La journée quotidienne de travail des reléguées est fixée à huit heures (de 7 heures à 11 heures et de 13 heures à 17 heures) et s'effectue dans un des quatre ateliers des travaux dirigés chacun par une sœur. Le réveil a lieu à 5 heures 45 et à leur levé les femmes effectuent leur prière en commun puis nettoient leur dortoir ainsi que leur atelier. Après avoir terminé le ménage, elles peuvent se promener dans la cour du dépôt en attendant l'heure du déjeuner. Celles qui peuvent s'acquitter de 10 centimes reçoivent un café et les autres doivent se contenter " d'une maigre soupe ».

À 7 heures, la cloche sonne et annonce le travail. Le silence le plus rigoureux est alors exigé et seuls les chants et les prières accompagnent le labeur. Le silence n'est rompu qu'à 11 heures, au moment de la distribution du premier repas. À 16 heures 30, le travail cesse et une demi-heure plus tard les reléguées soupent puis se voient accorder un moment de récréation. À 18 heures 30, les sœurs leur donnent l'ordre de regagner leur dortoir où elles récitent une dernière prière en commun avant de se coucher. Le silence total est alors à nouveau exigé. Ces femmes subissent ainsi un régime d'internement calqué sur les règles monacales de l'ordre des sœurs de Saint-Joseph de Cluny. Leur régime n'est donc en rien comparable à celui prévu par la loi sur la relégation, c'est-à-dire avec celui en cours à Saint-Jean. Les relégués y bénéficient de cases collectives de quarante places chacune où la surveillance n'est pas assurée la nuit. Dans ces chambrées, ils s'organisent pour mettre en place des activités lucratives et peuvent s'adonner à des loisirs. Chaque case comporte ainsi un rade, c'est-à-dire une échoppe tenue par un relégué qui y vend de l'alcool ou des denrées rares, comme le tabac. La nuit est ainsi souvent le prétexte à des parties de Marseillaise (jeu de cartes) 
qui égaient un peu le quotidien des forçats. Rien de comparable pour les reléguées qui sont soumises jusque dans leur intimité à une surveillance constante et étroite de la part des sœurs.

L'activité des reléguées est essentiellement constituée par des travaux de couture à destination des forçats. L'atelier de couture peut contenir jusqu'à 150 ouvrières qui sont employées à la confection de vêtements pour les relégués et les transportés, à la fabrication de moustiquaires et à toutes sortes de travaux de raccommodage du linge. Pour ces travaux, les reléguées gagnent 0,50 franc quotidien. Sur cette somme, 0,10 franc est versé à l'État, 0,20 franc est versé à leur pécule de réserve et 0,20 franc est versé à leur pécule disponible. Leur pécule disponible ne peut être dépensé qu'à la cantine administrative du dépôt qui est tenue par les sœurs tandis que leur pécule de réserve ne leur est remis qu'en cas de classement à la relégation individuelle. Ces faibles salaires ne leur permettent pas ainsi d'améliorer leur ordinaire. À l'inverse, et bien qu'ils disposent des mêmes salaires, l'organisation du travail des relégués collectifs internés à Saint-Jean est totalement différente. Ces derniers travaillent essentiellement le matin et le plus souvent sur des chantiers extérieurs. Ils peuvent ainsi consacrer leur après-midi à chasser ou à cultiver un lopin de terre et revendre leur production au personnel administratif. De plus, les métiers disponibles sont plus variés et permettent à leurs détenteurs de soutirer certains avantages dont ne peuvent bénéficier les reléguées. Les postes de cuisiniers, de boulangers, d'infirmiers ou bien de porte-clef (auxiliaires des surveillants militaires) permettent de soutirer facilement nourriture ou médicaments. Ces trafics, très courants au bagne et intitulés débrouille, autorisent ceux qui en bénéficient à gagner l'argent nécessaire à l'achat de denrées illégales ou bien les faveurs d'un môme (prostitué). Il permet également de se procurer le matériel nécessaire à une évasion. Rien de comparable ici encore avec le traitement réservé aux femmes qui les cantonnent exclusivement à des travaux de couture dont elles ne peuvent soutirer aucun avantage parallèle.

Mais les sœurs savent également faire montre de solidarité envers les reléguées. En 1903, le procureur de la République relève ainsi lors d'une visite du dépôt de nombreuses irrégularités quant à la gestion du travail des reléguées. Les religieuses revendent par exemple des denrées de la cantine à des personnes extérieures, disposent "indûment» du pécule des condamnées et vendent au profit de la communauté de menus travaux exécutés par des reléguées. De même, les sœurs servent parfois d'intermédiaires entre des reléguées et des personnes extérieures au dépôt. Elles revendent ainsi des objets confectionnés par des reléguées et partagent ensuite le fruit de la vente. Cet argent est le plus souvent redistribué par les sœurs aux reléguées âgées ou malades qui ne peuvent plus travailler et par là gagner un salaire leur permettant d'améliorer leur pécule. Ce surplus dégagé permet également aux sœurs de distribuer des gratifications supplémentaires aux ouvrières les plus méritantes et de distribuer également des petites sommes les jours de fêtes religieuses. En outre, la sœur supérieure se montre particulièrement laxiste en ce qui concerne les courriers que les reléguées adressent à leurs familles. Elle autorise ou ne censure pas la plupart d'entre eux alors que certains contiennent des critiques adressées à l'encontre de l'administration pénitentiaire ${ }^{18}$. Ces petits arrangements, bien qu'ils ne paraissent pas bien conséquents, agacent profondément le directeur de l'administration pénitentiaire. Pour ce dernier, le travail des reléguées doit être exclusivement orienté vers la production d'effets à destination de la population pénale. Le contexte de laïcisation et le débat autour de la séparation de l'Église et de l'État n'est guère à l'avantage des 
sœurs qui craignent à cette époque pour leur maintien au Maroni. L'affaire prend une telle ampleur que le directeur de l'administration pénitentiaire exige et obtient le remplacement au mois de mai 1905 de la sœur supérieure du dépôt ${ }^{19}$.

\section{Un régime disciplinaire drastique et une existence austère}

La monotonie de leur existence, de l'aveu même des sœurs, pèse sur ces femmes. Elle n'est rompue que le dimanche qui est jour de repos au dépôt. Le matin, les reléguées ont la possibilité de se rendre à la messe qui est célébrée à 7 heures 30 et peuvent ensuite établir leur correspondance et procéder au nettoyage de leurs effets personnels. L'après-midi, de 16 heures 30 à 18 heures, et uniquement lorsque le temps le permet, les reléguées toutes habillées d'un uniforme bleu ont droit à une promenade en dehors du dépôt sous la surveillance des sœurs. Défilant à travers Saint-Laurent, c'est le plus souvent à l'occasion de ces promenades qu'un forçat concessionnaire repère l'une d'elles et peut ensuite établir une demande de mariage auprès de la sœur supérieure. Mais en 1900, profitant de l'absence pour un congé en métropole du directeur de l'administration pénitentiaire, le commandant supérieur de la relégation les autorise à se rendre ponctuellement hors du dépôt soit pour se promener, soit pour trouver un engagement de travail. Au grand dam des sœurs, des reléguées reçoivent ainsi des autorisations ponctuelles de sortie qui se soldent pour la plupart par un encouragement à "l'immoralitée ${ }^{20}$ ». Les reléguées se faisant remarquer d'après les sœurs à l'extérieur "d'une façon tout à fait dégoûtante ${ }^{21}$ ». Suite aux plaintes de la sœur supérieure, le gouverneur abolit rapidement ces autorisations. Mais l'affaire prend une telle tournure qu'au mois de janvier 1902 la direction du dépôt des reléguées est officiellement retirée au commandant supérieur de la relégation et est désormais exclusivement confiée au commandant du dépôt de la transportation de Saint-Laurent.

De même, à sa prise de fonction au dépôt en 1893, la nouvelle sœur supérieure découvre avec horreur que les sœurs organisent des jeux et des danses avec les reléguées. Ces dernières, "dégradées plus bas que des transportés ${ }^{22}$ ", se déguisent en homme et dansent dans la cour du dépôt. La sœur supérieure met alors immédiatement fin à ces jeux. Les reléguées sont ainsi soumises à un régime disciplinaire bien plus draconien que celui subi par les relégués internés au dépôt de Saint-Jean. Elles ne peuvent pas sortir du couvent et sont maintenues en permanence sous la surveillance des sœurs. À l'inverse, les relégués disposent à Saint-Jean de refuges comme le théâtre de la relégation, la bibliothèque ou la fanfare au sein desquels ils peuvent s'affranchir un temps du régime du bagne. Ils peuvent également sortir du pénitencier qui n'est pas enclôt ou bien rendre visite à des camarades au sein d'autres cases. Les reléguées ne disposent pas d'une telle latitude et sont soumises à un régime disciplinaire drastique. Elles sont ainsi souvent déçues de leur sort à la relégation. Car la plupart s'imaginent avant leur départ pour la Guyane que la relégation s'apparente à un régime de liberté sous condition d'exil. L'enfermement qu'elles subissent au dépôt de Saint-Laurent est donc vécu avec un profond sentiment d'injustice :

Puis elles [les reléguées] attendaient une liberté relative qui ne leur est pas accordée de sorte que ces pauvres créatures souffrent et font souffrir les sœurs qui sont obligées de rester avec elles et de supporter leurs saillies de caractère qui sont loin d'être agréables ${ }^{23}$. 
Beaucoup tentent alors de s'évader car le dépôt se situe au centre de Saint-Laurent et est simplement borné par une barrière en bois facilement franchissable. Mais le directeur de l'administration pénitentiaire affirme que neuf fois sur dix les reléguées évadées sont ramenées par la police du Maroni après s'être signalées par « des scènes plus ou moins scandaleuses provoquées par l'ivresse ${ }^{24}$. » De 1887 au 31 décembre 1903, seules trois reléguées parviennent à s'évader et ne sont pas reprises. Pour réduire les résistances et soumettre les plus récalcitrantes, le dépôt comprend un local disciplinaire contenant douze cellules. Les reléguées qui y sont enfermées sont autorisées à demeurer dans une cour la journée et sont internées en cellule la nuit. Ce local disciplinaire s'apparente à « une prison sordide, mal enclos par une barrière en bois, des cours étroites où les femmes aux heures de repos et d'ombre ont peine à trouver une place suffisante ${ }^{25}$ ». Les reléguées punies de cellule sont mises au pain sec et à l'eau un jour sur trois et celles punies de cachot (bien que le quartier disciplinaire n'en soit pas pourvu) deux jours sur trois. Lorsqu'il se rend en inspection au dépôt des reléguées au mois de novembre 1896, le procureur de la République note en outre de nombreuses irrégularités au sein de ce quartier. Par exemple, le médecin ne visite les femmes enfermées en cellule que lorsque la sœur supérieure le lui demande. Alors que le décret du 22 août 1887 lui prescrit de visiter obligatoirement les cellules de la relégation tous les quinze jours et d'adresser un rapport au gouverneur après chaque visite. La sœur supérieure affirme également au procureur qu'elle possède des pouvoirs en matière disciplinaire et qu'elle peut infliger jusqu'à quatre nuits de prison. Pourtant le règlement du 25 septembre 1890 indique que les punitions ne peuvent être décidées que par une commission disciplinaire présidée par le commandant du dépôt de SaintLaurent. La sœur supérieure dispose seulement de la faculté d'assister aux sessions de cette commission mais n'a absolument aucun droit en matière disciplinaire. Néanmoins, la sœur supérieure recourt assez souvent de son propre chef à la punition du cachot :

Il faudrait être partout à la fois car il y a toujours ou une femme qui veut parler, ou une autre qui veut se disputer ou une autre qu'il faut menacer et même conduire au cachot pour la calmer et empêcher une bataille. C'est notre vie à toutes et tous les jours. Il est difficile de faire quelque bien à ces âmes, nous les voyons avec peine, n'aspirer après la liberté que pour se livrer à la plus honteuse prostitution. Ici, la seule consolation que nous ayons, c'est d'empêcher peut-être quelques péchés ${ }^{26}$.

31 La promiscuité et la stricte discipline auxquelles sont soumises ces femmes entraînent effectivement de nombreuses tensions et des disputes entre elles. Placées sous la tutelle des sœurs qui les punissent au moindre manquement, ces dernières n'envisagent en outre leur sortie du couvent que comme un probable encouragement à l'immoralité. La représentation nourrie à l'encontre de ces femmes alterne ainsi entre épouses et prostituées. Leur rôle se cantonne à ces deux extrémités et elles sont les victimes d'une domination où percent tous les stéréotypes d'une société masculine ${ }^{27}$. Saisies par leurs corps "hystérisés ${ }^{28}$ » dont elles ne peuvent pas intégralement disposer, elles sont regardées comme des " pécheresses " susceptibles de troubler par leur immoralité une colonie de peuplement encore à ses balbutiements. Il appartient donc de les maintenir sous une discipline inflexible, seule à même de réprimer leurs penchants "vicieux ». Et ce afin de les plier aux rôles dévolus à leur sexe, c'est-à-dire ceux d'épouses et de mères nourricières. 


\section{Les maladies et le sort des reléguées individuelles}

Les reléguées connaissent un taux de mortalité très élevé. Mais les terribles épidémies qu'elles essuient périodiquement émeuvent peu les autorités pénitentiaires. Ainsi, à la fin du mois d'avril 1891 et jusqu'au mois de février 1892, une épidémie de «fièvre bilieuse » sévit parmi les reléguées. Ces dernières sont quasiment toutes atteintes par le mal et certaines connaissent jusqu'à quinze à vingt rechutes. Durant cette épidémie, l'atelier est totalement désert et le dortoir est reconverti en infirmerie. Les sœurs sont totalement débordées par le nombre de malades et l'administration pénitentiaire ne leur vient absolument pas en aide. Non seulement les femmes atteintes sont trop nombreuses pour être envoyées à l'hôpital de Saint-Laurent mais elles ne reçoivent de plus aucun médicament. Les sœurs achètent sur leurs propres fonds et ce durant plusieurs mois la quinine et les médicaments nécessaires à leur rétablissement. La mère supérieure fait distribuer quotidiennement aux plus souffrantes quelques œufs, un peu de rhum et quelques morceaux de pain blanc. En tout, vingt femmes décèdent durant cette première épidémie. En matière de santé, comme dans la plupart des autres chapitres, l'administration pénitentiaire se repose ainsi sur la seule initiative et sur les fonds propres des sœurs. Par exemple, en 1900, une épidémie de grippe sévit à SaintLaurent et 93 reléguées en sont atteintes. Les sœurs sont à nouveau littéralement débordées par la situation sanitaire du dépôt. Face à cette situation, le directeur de l'administration pénitentiaire consent à «un acte d'humanité » et fournit les médicaments et les vivres nécessaires pour faire face à l'épidémie. Mais cet acte lui semble plus dicté par la demande pressante des sœurs que par un quelconque intérêt pour le sort des reléguées:

M. le Directeur ayant été informé de cet état de choses [l'épidémie de grippe], s'est rendu sur les lieux et ordonné qu'il soit mis à notre disposition outre les médicaments, du café, du lait et du vin pour distribuer aux malades selon le besoin pendant un mois. Cet acte d'humanité de la part de ce haut fonctionnaire a produit un grand bien sur le moral de ces malheureuses exilées qui excitent rarement la pitié de quelqu'un qui les connaît. Elles ignorent que dans cette circonstance $\mathrm{M}$. le Directeur a agi de la sorte pour nous faire plaisir et nous procurer les moyens de soulager des misères qui nous auraient navré le cœur ; car ces femmes ne pouvant faire leur tâche ni manger la nourriture grossière qui leur est donnée, il nous aurait été bien dur de les voir souffrir et en même temps difficile de faire la charité à tant de malades à la fois vu que nous la faisons en tout temps aux plus nécessiteuses ${ }^{29}$.

Les reléguées sont victimes d'épidémies de fièvre jaune, de dysenterie et de paludisme qui s'abattent régulièrement sur la région du Maroni. De 1887 au 31 décembre 1903, la Guyane reçoit près de 499 reléguées et sur ce nombre près de 267 décèdent dans la colonie, soit $53,50 \%$ de l'effectif total. Le taux de mortalité moyen annuel s'élève ainsi à $8,46 \%$ de l'effectif:

\begin{tabular}{|l|l|l|l|}
\hline Années & Effectif au 31 décembre & Nombre de décès & Proportion pour 100 \\
\hline 1887 & 48 & 0 & 0 \\
\hline 1888 & 93 & 9 & 9,67 \\
\hline 1889 & 140 & 8 & 5,71 \\
\hline
\end{tabular}




\begin{tabular}{|l|l|l|l|}
\hline 1890 & 152 & 6 & 3,94 \\
\hline 1891 & 154 & 20 & 12,98 \\
\hline 1892 & 166 & 19 & 11,44 \\
\hline 1893 & 158 & 8 & 5,06 \\
\hline 1894 & 165 & 9 & 5,45 \\
\hline 1895 & 179 & 6 & 3,35 \\
\hline 1896 & 186 & 9 & 4,83 \\
\hline 1897 & 220 & 6 & 2,72 \\
\hline 1898 & 257 & 10 & 3,89 \\
\hline 1899 & 261 & 16 & 6,13 \\
\hline 1900 & 270 & 24 & 8,88 \\
\hline 1901 & 275 & 58 & 10,18 \\
\hline 1902 & 242 & 28,80 \\
\hline 1903 & 223 & 58 & 26 \\
\hline
\end{tabular}

Mais les femmes placées en relégation individuelle connaissent en moyenne un taux de mortalité plus élevé que celles internées au dépôt :

\begin{tabular}{|l|c|l|}
\hline Taux de mortalité des reléguées collectives et des reléguées individuelles & 1902 & 1903 \\
\hline Effectif moyen des reléguées collectives & 135 & 124 \\
\hline Décès des reléguées collectives & 14 & 24 \\
\hline Proportion pour 100 & 10,4 & 19,3 \\
\hline Effectif moyen des reléguées individuelles & 124 & 231 \\
\hline Décès des reléguées individuelles & 17 & 58 \\
\hline Proportion pour 100 & 13,7 & 25,1 \\
\hline
\end{tabular}

35 La mortalité élevée des reléguées individuelles s'explique par plusieurs facteurs. Elles sont tout d'abord durement concurrencées par leurs homologues collectives qui effectuent au sein du dépôt nombre de petits travaux (comme des travaux de couture ou de nettoyage du linge) pour des particuliers ou des membres de l'administration pénitentiaire. Elles parviennent ainsi difficilement à trouver un emploi et sont de plus 
pour la plupart interdites de séjour à Cayenne. Le séjour du chef-lieu de la colonie, pourtant le plus susceptible de les absorber économiquement, leur étant interdit, elles sont donc tenues de résider à Saint-Laurent où elles sont concurrencées par les reléguées collectives. Beaucoup sombrent alors dans un sort de misère. Ou bien profitent de leur placement à la relégation individuelle pour dépenser intégralement leur pécule de réserve puis réintègrent le dépôt :

Étant donné la situation actuelle au Maroni, on peut avancer, à mon avis, que la relégation individuelle a été appliquée aux femmes sur une trop grande échelle. Celles de ces femmes qui se conduisent le mieux vivent en concubinage, et les autres se procurent, par la prostitution, les ressources qui leur sont indispensables ${ }^{30}$.

Les reléguées individuelles sont en outre beaucoup plus exposées aux rigueurs du climat guyanais que les reléguées collectives. Ces dernières vivent au dépôt où les sœurs leur dispensent des soins à l'infirmerie et leur assurent le gîte et le couvert. Les reléguées individuelles doivent se débrouiller comme elles peuvent et la plupart, épuisées et malades, se présentent trop tardivement à l'hôpital de Saint-Laurent pour recevoir des soins. Par exemple, le nombre de reléguées collectives entrées à l'hôpital en 1903 s'élève à $35,3 \%$ alors que cette proportion est de seulement $16,4 \%$ pour les reléguées individuelles bien que leur taux de mortalité est plus élevé. Leur situation est si dramatique que la même année le directeur de l'administration pénitentiaire décide de ne plus accorder de demande de mise en relégation individuelle et intime l'ordre aux sœurs de n'employer les reléguées qu'à la confection exclusive de vêtements à destination de la population pénale. Désormais, pour échapper au couvent, il ne leur reste plus d'autre opportunité que de se placer sous la domination d'un époux.

\section{Les mariages à la relégation}

Théoriquement, un relégué placé en relégation individuelle peut être rejoint par son épouse et ses enfants demeurés en métropole. Mais en règle générale rares sont les épouses qui ont la patience d'attendre le changement de statut de leur conjoint interné en Guyane. De plus, ces derniers parviennent rarement à se suffire à eux-mêmes à leur sortie du pénitencier. La relégation étant une peine perpétuelle accomplie sur un territoire peu engageant, la plupart des épouses de relégués restées en métropole préfèrent divorcer :

René,

Je fais réponse à ta lettre avec du retard certes c'est vrai, mais je n'ai pas pu faire autrement. Tu me dis que ma réponse sera définitive donc René écoute bien : tu me dis que je ne veux pas divorcer car je suis très chrétienne, et bien non je ne [le] suis plus, je ne crois plus en rien car voilà je n'avais jamais fait de mal à personne et sans pitié ma vie a été gâchée. Je me suis retrouvée avec mes deux petits sans travail à l'hôtel. D'ailleurs je ne sais pas pourquoi je te dis tout cela car tu le sais mais c'est pour bien te faire comprendre ma résolution. Je suis depuis bientôt deux ans avec un brave garçon qui, sans me demander des explications, m'a prise avec mes deux petits et depuis il travaille pour les nourrir. Je lui dois tout. Alors René je vais divorcer, je vais me refaire une vie avec cet homme qui m'aime sincèrement car il me l'a prouvé. Il aime les petits comme s'ils étaient à lui et les enfants [le] lui rendent bien. Donc ma résolution est prise, je demande le divorce. Maintenant je te donne des nouvelles de [...]. Les petits sont très bien, je les ai vus il y a trois semaines, on allait à l'enterrement de papa. Oui papa est mort, il avait pourtant jamais fait de mal à personne lui non plus, que du bien au contraire. Et tu crois 
encore qu'il y a un Dieu toi. S'il y en avait eu un, il ne nous l'aurait pas pris à 54 ans. Raymonde elle est mariée et elle a un petit garçon. Chez Titin, c'est toujours pareil. Enfin, revenons à nous. Tu le dis toi-même sur ta lettre, à ta libération la vie commune ne serait plus possible, alors ne m'en veux pas, on n'était plus faits pour vivre ensemble. Tu vois que je suis franche, tu ne veux pas d'équivoques entre nous, il n'y en aura pas. Donc René par cette lettre je te dis adieu.

Ginette $^{31}$. doivent s'organiser pour survivre au départ d'un père et d'un mari souvent soutien de famille. Certaines épouses promettent pourtant à leurs maris de les rejoindre au bagne :

Mon amour adoré,

Avant que je puisse aller te voir, je viens par cette lettre te souhaiter mes meilleurs vœux de santé et une meilleure année que celle que nous venons de finir pour tous les deux. Oui mon Antoine, ayons du courage et surtout aimons-nous bien et tu verras que nous aurons une meilleure année devant nous. Dans mes prières, il y a toujours une pensée pour toi. Tous mes baisers pour toi. Je t'embrasse. Comme je t'aime $^{32}$.

'envoi de femmes au bagne participe dans l'esprit du législateur à l'effort colonial assorti à l'installation des relégués en Guyane. En plus de pouvoir obtenir une concession de terre, le relégué a également la possibilité de contracter un mariage et de fonder une famille. Mais à la suite de la première demande de mariage adressée par un relégué collectif en 1889 , et devant l'absence de règlement déterminant les conditions dans lesquelles les relégués peuvent se marier, le ministre des Colonies décide de limiter le droit au mariage aux seuls relégués individuels. Les relégués collectifs, pourtant les plus nombreux en Guyane, ne peuvent donc pas se marier et aucun d'entre eux ne peut fonder de famille à Saint-Jean. D'autre part, leur nombre et l'âge de ces femmes rendent en définitive cette expérience totalement marginale. À peine 519 reléguées débarquent en Guyane de 1887 à 1904 et leur âge moyen au moment de leur condamnation à la relégation est de 38 ans :

Ce n'est pas avec les femmes reléguées que l'on pourra arriver à constituer des familles dans les colonies pénales ${ }^{33}$.

Les reléguées rencontrent également de leur côté beaucoup de difficultés pour se marier. C'est le cas par exemple de Philomène Petit (244) qui s'évade du dépôt en 1896 pour rejoindre son amant et qui est punie d'un mois de cachot car les sœurs ne l'autorisent pas à avoir des entrevues avec un "Européen ». La sœur supérieure est effectivement hostile aux mariages entre des reléguées et des relégués européens et préfère favoriser des unions avec des Maghrébins. D'après elle, les unions avec les Européens tournent rapidement court. Mais même auprès de condamnés d'origine maghrébine, la sœur supérieure se montre très sceptique sur ces mariages et ne les accepte qu'au compte-gouttes :

Ces jours derniers, 6 de nos femmes se sont mariées à des concessionnaires, 8 autres se préparent à cet acte plus ou moins risqué ; bien souvent c'est pour sortir du Dépôt qu'elles se marient, se réservant d'agir ensuite à leur guise ; et en effet il y en a plus de la moitié qui ne restent pas avec leur mari légitime; chose bien déplorable ${ }^{34}$.

41 D'après la sœur supérieure, la plupart des femmes qui se marient se conduisent particulièrement mal à leur sortie du dépôt. Les mariages représentent seulement pour elles l'occasion d'épuiser "leur pécule à boire et à faire la noce ${ }^{35}$ ». La plupart abandonnent ensuite leur mari et réintègrent le dépôt une fois leurs ressources 
épuisées. Mais d'autres peuvent également être prostituées et maltraitées par leur mari. C'est le cas notamment d'Antoinette Ximénes qui est mariée au libéré concessionnaire Mohamed Ali Haddad. Au mois de novembre 1902, brutalisée et prostituée par son mari, Antoinette quitte le domicile conjugal et demande à se séparer de son mari :

Monsieur le Commandant,

C'est avec le plus grand respect que je m'adresse à votre bienveillance pour vous adresser la réclamation suivante. Depuis quelque temps, je suis très malheureuse avec mon mari, tous les jours des scènes à n'en plus finir, je travaille moi comme une pauvre esclave quant à lui la moitié du temps il ne travaille pas. Hier au soir il m'a mise à la porte et j'ai été forcée de me coucher dans la canne, ceci arrive très souvent, mais je commence à me fatiguer de cette vie que cet homme me fait faire. C'est pour cela Monsieur le Commandant que je voudrais quitter mon mari et travailler pour moi-même ${ }^{36} »$.

Le surveillant chargé des concessions à Saint-Maurice confirme l'état déplorable du couple et souligne qu'Antoinette se livre en effet à la prostitution pour faire vivre son mari :

La relégué individuelle Ximénes Antoinette, femme du libéré concessionnaire $\mathrm{n}^{\circ}$ mle 6073 Mohamed Ali [...] n'a pas réintégré le domicile. Le mariage est très mauvais. La femme est ivrogne et le mari paresseux, ne travaillent presque jamais. Vit de la prostitution de la femme ${ }^{37}$.

Les cas de prostitution de reléguées établies en mariage légitime ou bien établies en union libre à Saint-Laurent sont courants et ces femmes sont tout simplement exploitées par leurs conjoints ou par leurs maris. Mais la prostitution peut également être la dernière extrémité pour certaines reléguées individuelles qui s'y livrent afin de survivre aux situations de misère économique qu'elles connaissent à leur sortie du dépôt :

Notre œuvre touchant les femmes reléguées subit un mouvement sensible, 13 mariages ont été célébrés à l'édification de la paroisse, 17 autres sont en projet. Beaucoup d'autres femmes ont obtenu une liberté relative, l'individuelle. Malheureusement, la plupart de ces dernières se mettent en ménage illégitime; d'autres mènent une vie plus scandaleuse encore de sorte que ces malheureuses vont continuer dans ce pays le triste métier qui leur a valu l'exil et attireront sur le pays la malédiction de Dieu ${ }^{38}$.

Outre la prostitution, les reléguées peuvent également être victimes de maris qui les brutalisent. Le transporté Belkassen Ben Bou Dam a été séparé et éloigné de sa femme par l'administration pénitentiaire du fait des brutalités et des mauvais traitements qu'il lui infligeait. En 1896, cette dernière exploite seule leur concession mais son mari demande à nouveau à la revoir :

Monsieur le Surveillant principal,

En réponse à votre note de ce jour. J'ai l'honneur de vous faire connaître que la femme Rébaïa Bent Charif (320) ne veut en aucune façon entendre parler de son mari, Belkassen Ben Bou Dam, envoyé aux îles du Salut par mesure de sûreté. Elle préfère tout abandonner et rentrer au couvent plutôt que de se voir réduite à être obligée de revivre avec Belkassen. Il a failli, d'après elle, deux fois la tuer et elle ne veut plus s'exposer à un semblable danger. Elle implore l'Administration de maintenir les décisions prises à ce sujet ${ }^{39}$.

Pour toutes ces raisons, la sœur supérieure autorise peu d'unions ce qui entraîne de nombreuses protestations de la part des reléguées. Le procureur de la République, lors de sa tournée d'inspection du couvent au mois de novembre 1896, reçoit ainsi des 
plaintes de neuf reléguées qui ont sollicité en vain une demande de mariage. Non seulement la sœur supérieure suspend leur demande mais les empêche de plus d'avoir des entretiens avec leurs fiancés. Car une fois par semaine a lieu sous la surveillance des sœurs des entrevues au parloir du dépôt. Un forçat libéré nanti d'une autorisation du directeur de l'administration pénitentiaire se rend auprès de la sœur supérieure et lui demande de lui faire rencontrer une reléguée. Le forçat libéré peut demander à rencontrer précisément une condamnée ou bien peut laisser le soin à la sœur supérieure de la sélectionner pour lui. Après avoir obtenu l'autorisation de se présenter au parloir, le demandeur est ensuite prié de décliner l'identité de sa future promise. La démarche du relégué individuel Jules Julien (5975), engagé en tant que cuisinier auprès d'un particulier de Saint-Laurent, est de ce point de vue assez saisissante :

Expose à Monsieur le Commandant Supérieur qu'il a été informé par M. le Commissaire de police que sa demande de parloir avait reçu un accueil favorable auprès de vous. Il y manquait la désignation de la personne avec laquelle je désire convoler légitimement. Sur ce point, je ne peux être fixé, n'ayant jamais, jusqu'à ce jour, eu l'occasion de remarquer telle ou telle. Mon idée de mariage est, en raison de ma profession, de trouver une femme de ménage, voilà simplement mon but. Laquelle? Je ne pourrai la faire connaître qu'avec l'autorisation de Monsieur le Commandant Supérieur lorsqu'il m'aura préalablement donné l'agrément de me présenter au parloir du couvent, auprès de madame la Supérieure, muni d'une permission régulière que j'ose solliciter de la haute bienveillance de Monsieur le Commandant Supérieur.

Julien Jules ${ }^{40}$.

Une fois la promise désignée, la sœur supérieure aménage ensuite la rencontre qui a lieu au parloir du dépôt sous la surveillance d'une sœur " portière ». Si l'entretien est concluant, les deux fiancés se voient tous les quinze jours jusqu'à la célébration de leur mariage qui est décidé entre eux et la sœur supérieure. Mais outre l'aspect strictement sentimental, le mariage représente surtout pour les reléguées l'unique possibilité d'échapper au régime collectif du dépôt. C'est pour cela que beaucoup cherchent des unions qui ne les engagent en rien, délaissant vite leurs maris et savourant enfin leur liberté retrouvée :

Le rêve de la reléguée est d'être tirée du couvent par le mariage. Cela se présente quelquefois. Ceux qui donnent le titre d'épouses à ces malheureuses, pour la plupart laides et sans âges, sont des Arabes concessionnaires ou libérés. [...] Les Algériens qui convolent dans ces conditions sont sganarellisés [sic] sans vergogne; ils se vengent de leurs malheurs conjugaux en reconduisant au couvent les femmes qu'ils en ont tirées et qui n'en sortiront que sur la demande expresse du mari ${ }^{41}$.

Car les réintégrations au dépôt collectif à la demande de maris de reléguées sont relativement fréquentes. Ces derniers possèdent grâce à ce moyen un ascendant qui leur permet de soumettre leurs épouses et peuvent les punir en cas d'inconduite. Marié à la reléguée individuelle Marie Chrétien (414), le transporté concessionnaire JeanMarie Godefroy se rend au mois d'octobre 1902 au commissariat pour réclamer la réintégration au dépôt de sa femme. Il se plaint qu'en son absence elle se livre à la prostitution et ne s'occupe pas de son ménage. Réintégrée immédiatement au dépôt, Jean-Marie Godefroy propose au mois de février 1903 de la reprendre à ses côtés en espérant l'avoir corrigée de ses mauvais penchants :

Monsieur le Commandant,

Je viens humblement vous prier de vouloir bien m'autoriser à reprendre ma femme, la nommée Chrétien mle. 414, que j'ai fait réintégrer il y a environ 3 mois. J'espère que la mesure que j'avais fait prendre contre elle l'aura corrigée. Dans l'espoir 
d'une réponse favorable à la présente demande, daignez agréer, Monsieur le

Commandant, mes sentiments respectueux ${ }^{42}$. " chefs de famille». Au moindre écart, le mari éconduit peut priver son épouse de sa liberté, user de son droit de correction "paternel» et la remettre à la discipline inflexible du couvent. L'époux demeure ainsi le garant du bon ordre et de la moralité de son ménage et est le seul juge de la conduite de sa femme ${ }^{43}$. Les reléguées sont ainsi transbahutées de la domination des sœurs à celle de leurs époux, et vice versa. La plupart de ces mariages ne donnent en outre guère de résultats car ces femmes, trop peu nombreuses et trop âgées, subissent ainsi que leurs enfants un taux de mortalité très important. En 1905, date à laquelle le ministère des Colonies décide de ne plus envoyer de reléguées en Guyane, le nombre de mariages pénaux s'élève tout juste à 103 et les épouses sont toutes âgées en moyenne de 40 à 50 ans.

\section{Une abolition longue et tardive}

Au début de l'année 1904, face au taux de mortalité très important rencontré chez les reléguées en 1902 et en 1903, le directeur de l'administration pénitentiaire propose au gouverneur de rendre la relégation des femmes facultative comme pour les transportées. Ému par le sort de ces femmes, le ministre des Colonies se range à son avis et abolit au mois d'octobre 1905 la relégation pour les femmes :

Mon attention a été appelée sur les conditions rigoureuses imposées aux femmes récidivistes par leur envoi dans les Colonies pénitentiaires, contrairement au traitement appliqué aux condamnées aux travaux forcés, qui, bien que frappées d'une peine plus élevée, sont maintenues en France et subissent leur détention dans les maisons centrales de la métropole. En vue de remédier à la situation défavorable créée par le législateur de 1885 aux femmes récidivistes et dont l'opinion publique s'est émue dans ces derniers temps, j'ai préparé le projet de loi, ci-joint, tendant à la suppression de la relégation aux colonies des femmes récidivistes ${ }^{44}$.

De son côté, le garde des Sceaux se montre favorable à ce projet et souligne l'échec total de la relégation des femmes en Guyane qui «n'a pas produit d'effets heureux ${ }^{45}$ » ainsi que sa disproportion au vu du sort des transportées. De plus, l'envoi des reléguées en Guyane est devenu totalement marginal: 18 à peine y sont envoyées en 1903 et seulement 7 en 1904. Ces faibles chiffres sont dus en partie à l'obstruction organisée par la commission de classement des récidivistes ${ }^{46}$ en métropole. Ses membres, "par horreur de se faire bourreaux ${ }^{47}$ ", prolongent sciemment la détention des reléguées afin de les empêcher d'embarquer pour la Guyane.

51 Mais il faut toutefois attendre 1907 pour que la relégation des femmes soit enfin abolie grâce à la loi du 19 juillet 1907. Dorénavant, l'article 6 de la loi du 27 mai 1885 stipule que la relégation « n'est plus applicable aux femmes ni aux individus qui seraient âgés de plus de soixante ans ou de moins de vingt ans à l'expiration de leur peine ». Elle est remplacée pour les femmes par une interdiction de séjour de vingt ans. Cette loi permet de ne plus envoyer de reléguées en Guyane et son application autorise rétroactivement celles présentes dans la colonie à pouvoir rentrer en métropole. Au mois de septembre 1907, le garde des Sceaux ordonne donc la mise en liberté immédiate de toutes les reléguées internées au dépôt de Saint-Laurent. Mais le directeur de l'administration pénitentiaire, craignant pour la sécurité publique de la colonie, parvient à obtenir du ministre des Colonies la suspension du décret d'application en Guyane de la loi du 19

Crime, Histoire \& Sociétés / Crime, History \& Societies, Vol. 17, $\mathrm{n}^{\circ} 1$ | 2013 
juillet 1907. De ce fait, le ministre des Colonies n'autorise pas leur libération effective et ne permet le retour qu'à celles qui ont les moyens de couvrir les frais de leur rapatriement.

En parallèle, toutes les femmes condamnées à la relégation en métropole sont désormais libres et sont seulement astreintes à une interdiction de séjour de vingt ans. Celles encore internées en Guyane doivent donc attendre la véritable abolition de la relégation qui n'intervient qu'avec la loi du 8 août 1913 et qui rend enfin applicable aux colonies la loi du 19 juillet 1907. À la suite de cette loi, l'ordre est donné au mois de février 1914 de libérer immédiatement toutes les reléguées du dépôt. Le ministre des Colonies s'empresse d'indiquer au gouverneur de la colonie que ces femmes peuvent si elles le désirent rentrer en métropole ou dans leurs colonies d'origine, mais toujours à leurs frais. Au mois de mars 1914, toutes les reléguées animées d'une " joie délirante " quittent le couvent et partent s'installer à Saint-Laurent. Il reste 73 femmes encore soumises à la relégation mais seulement 11 possèdent les fonds nécessaires pour payer leurs frais de passage. Les autres doivent donc rester dans la colonie où la plupart, trop âgées, sans emploi et interdites de séjour à Cayenne, ne tardent pas à réintégrer rapidement le dépôt :

Enfin, en conformité de vos instructions, cinq femmes hors d'état de pourvoir à leur entretien en raison de leur âge et de leurs infirmités, ont été conservées en subsistance au dépôt, sur leur demande. Mais leur nombre parait devoir s'augmenter, car la plupart des femmes mises en liberté ne paraissent pas capables de gagner leur vie par le travail en Guyane où cette main-d'œuvre peu appréciée ne trouvera point à s'employer ${ }^{48} »$.

53 Après sa fermeture, le dépôt est transformé en asile par les sœurs qui y accueillent toujours quelques femmes revenues d'elles-mêmes. Et ce jusqu'au $1^{\text {er }}$ juillet 1923, date à laquelle le conseil de la congrégation décide la suppression de l'œuvre des sœurs de Saint-Joseph de Cluny à Saint-Laurent et ordonne la fermeture définitive du dépôt.

\section{Conclusion}

54 L'histoire de la relégation des femmes illustre d'une manière saisissante le détournement de sens opéré par les autorités pénitentiaires dans l'application de cette mesure sur le sol de la Guyane. Elles ne devaient pas être des condamnées aux travaux forcés internées au sein d'un couvent mais des exilées soumises à une résidence perpétuelle. Car il est nécessaire de le souligner: les reléguées condamnées par les tribunaux français ont toutes déjà purgé leur peine principale sur le sol de la métropole ou de leurs colonies d'origine lorsqu'elles débarquent à Saint-Laurent. La relégation s'apparente néanmoins dans les faits à une seconde peine bien plus drastique que leur peine principale et ces femmes sont soumises à un régime disciplinaire d'une sévérité extrême. En outre, la direction de ces femmes a également été détournée au profit des sœurs de l'œuvre de Saint-Joseph de Cluny qui se sont arrogé la direction morale et physique des reléguées. Ces dernières représentent pour les sœurs supérieures un véritable enjeu de souveraineté. Et ces femmes se sont ainsi retrouvées inféodées aux règles austères d'un ordre religieux et sujettes à un pouvoir encadré par aucun texte légal, si ce n'est par l'arbitraire plus ou moins bienveillant des sœurs supérieures.

Soumises à un système qui les résume à des condamnées aux travaux forcés, les reléguées sont également les victimes d'une domination qui les réduit à de simples 
génitrices. La différence des sexes opérée en Guyane entre les hommes et les femmes relégués est clairement au désavantage de ces dernières. Pour espérer échapper au couvent, ces femmes n'ont pas d'autre espoir que de trouver un mari et de se plier à une économie domestique au sein de laquelle elles basculent sous la souveraineté de leurs époux. D'une domination l'autre, les reléguées demeurent des mineures ballottées entre le couvent et sa discipline inflexible et des maris qui peuvent souvent abuser d'elles.

\section{BIBLIOGRAPHIE}

Bard C., Chauvaud F., Perrot M., Petit J.-G., Femmes et justice pénale XIX ${ }^{e}-X X^{e}$ siècles, Rennes, Presses Universitaires de Rennes, 2002.

Bulletin de la Congrégation des Scurs de Saint-Joseph de Cluny, septembre 1891, XXIII.

Bourdieu P., La domination masculine, Paris, Seuil, 1998.

Devèze M., Déportés et bagnards, Paris, Julliard, 1965.

Donet-Vincent D., La fin du bagne, 1923-1953, Rennes, Éditions Ouest-France, 1992

Donet-Vincent D., De soleil et de silence. Histoire des bagnes de Guyane, Paris, La Boutique de

l'Histoire, 2003.

Foucault M., Histoire de la sexualité I. La volonté de savoir, Paris, Gallimard, 1976.

Krakovitch 0. , «Le bien d'autrui tu ne prendras... » ou à Cayenne pour le vol d'une paire de draps. L'envoi de femmes aux bagnes de Cayenne et de Nouvelle-Calédonie pour vols et escroqueries, de 1858 à 1883, Bulletin d'Information des Études Féminines, décembre 1983, 13, pp. 61-81.

Krakovitch O., Les femmes dans les bagnes de Guyane et de Nouvelle-Calédonie, Histoire de la justice, 1990, 3, pp. 51-68.

Krakovitch O., Lettres de bagnardes et prisonnières (1855-1890), Histoire de la Justice, 1992, 5, pp. 153-170.

Krakovitch O., Les femmes bagnardes, Paris, Perrin, 1998.

Liard-Courtois A., Souvenirs du bagne, Paris, Les Passés Simples, 2005.

Langlois $C$., L'introduction des congrégations féminines dans le système pénitentiaire français (1839-1880), in Petit J.-G. (dir.), La prison, le bagne et l'histoire, Genève, librairie des Méridiens, édition Médecine et Hygiène, 1984, pp. 129-140.

Michel P., Bagnards. La terre de la grande punition. Cayenne 1852-1953, Paris, Autrement, 2000.

Mimande P., Une loi cruelle. La relégation des femmes, Le Correspondant, 1905, 220, pp. 740- 758.

Notice sur la relégation à la Guyane française et à la Nouvelle-Calédonie, Paris, Imprimerie nationale, 1895-1911.

Redfield P., Space in the Tropics : From Convicts to Rockets in French Guiana, Berkeley, Los Angeles, London, University of California Press, 2000. 
Sanchez J.-L., La relégation (loi du 27 mai 1885), Criminocorpus, revue hypermédia [En ligne], Les bagnes coloniaux, Articles, mis en ligne le $1^{\mathrm{er}}$ janvier 2005, consulté le 2 juillet 2012. URL [http:// criminocorpus.revues.org/181].

Sanchez J.-L., La relégation des récidivistes en Guyane française. Les relégués au bagne colonial de SaintJean-du-Maroni, 1887-1953, thèse pour le doctorat d'histoire, sous la direction de Gérard Noiriel, Paris, École des Hautes Études en Sciences Sociales, 2009. En ligne [http://hal.archivesouvertes.fr/tel-00506778/ - lien valide le 2 juillet 2012].

Toth S. A., Beyond Papillon. The French Overseas Penal Colonies, 1854-1952, Lincoln, University of Nebraska Press, 2006

\section{Archives}

Archives de la congrégation de Saint-Joseph de Cluny, Paris.

Série Correspondance Maroni 1858-1923.

Série Cayenne, administration.

* 2 A I 5-1.

Série Saint-Laurent-du-Maroni.

* 2 A I 7.

Série Comptes rendus de visite.

*2 A I 5-10.

Série Pièces ministère.

* 5 A GUYA 3.

Série Situation du personnel, Guyane, 1862-1905.

Archives départementales de Guyane, Cayenne.

Série IX, Administration pénitentiaire.

Archives nationales, CARAN, Paris.

Série Division criminelle du ministère de la justice.

* BB 181804

Archives nationales d'outre-mer, Aix-en-Provence.

Série Colonies $\mathrm{H}$.

\section{NOTES}

2. Krakovitch (1990, p. 259).

3. Devèze (1965, p. 142).

4. Donet-Vincent $(1992,2003)$.

5. Redfield (2000); Toth (2006).

6. Sanchez (2005). 
7. Compte rendu de la visite de la Révérende-Mère supérieure principale dans les communautés et les maisons du district, District de la Guyane, 2 A i 5-10, Archives de la Congrégation des Sœurs de Saint-Joseph de Cluny (désormais ACSJC).

8. Langlois (1984, p. 136).

9. Bulletin de la Congrégation des Sours de Saint-Joseph de Cluny (1891, pp. 1055-1056).

10. C'est-à-dire les transportées. La sœur supérieure à la mère principale, le 28 octobre 1888, ACSJC.

11. La sœur supérieure à la mère principale, le $1^{\mathrm{er}}$ juin 1889, 2 A i7, ACSJC.

12. Ibid.

13. Ibid.

14. Ibid.

15. L'inspecteur général Espent au sous-secrétaire d'État des colonies, le 7 juin 1889, Archives Nationales d'Outre-Mer (désormais ANOM) H 1869.

16. L'inspecteur des colonies Picquié au ministre des Colonies, le 11 mars 1896, ANOM H 1870.

17. La sœur supérieure à la mère principale, le 14 juillet 1900, ACSJC.

18. La sœur supérieure à la mère principale, le 31 mars 1902, ACSJC.

19. La congrégation de Saint-Joseph de Cluny au ministre des Colonies, le 27 mai 1905, ANOM H 1862.

20. La sœur supérieure à la mère principale, le 5 février 1902, ACSJC.

21. La sœur supérieure à la mère principale, le 28 septembre 1900, ACSJC.

22. La sœur supérieure à la mère principale, le 24 mars 1893, ACSJC.

23. La sœur supérieure à la mère principale, le $1^{\text {er }}$ juin 1895 , ACSJC.

24. Rapport au gouverneur, le 19 mars 1904, Archives Départementales de Guyane (désormais $\mathrm{ADG})$

IX 26 bis.

25. L'inspecteur des colonies Picquié au ministre des Colonies, le 11 mars 1896, ANOM H 1870.

26. La sœur supérieure à la mère principale, le 11 septembre 1906, ACSJC.

27. Bard, Chauvaud, Perrot, Petit (2002, p. 25).

28. Foucault (1976, p. 137).

29. La sœur supérieure à la mère principale, le 8 mai 1900, ACSJC.

30. Rapport au gouverneur, le 19 mars 1904, ADG IX 26 bis.

31. Lettre adressée le 28 avril 1937, ANOM H 5154.

32. Carte écrite en décembre 1934 et trouvée dans le dossier individuel du relégué Charles Isidore Claude (16689), ANOM H 3842.

33. Notice sur la relégation à la Guyane française et à la Nouvelle-Calédonie (1890, p. 17).

34. La sœur supérieure à la mère principale, le 25 juin 1902, ACSJC.

35. Rapport d'inspection des établissements pénitentiaires par le chef du service judiciaire, le 30 septembre 1896, ANOM H 5151.

36. Antoinette Ximénes au commandant supérieur du dépôt de Saint-Laurent, le 8 novembre 1902, Musée National des Prisons (désormais MNP).

37. Le surveillant des concessions de Saint-Maurice au chef de camp, le 11 novembre 1902, MNP.

38. La sœur supérieure à la mère principale, le 12 juillet 1899, ACSJC.

39. Le surveillant chargé des concessions au surveillant principal, le 22 septembre 1896, MNP.

40. Jules Julien au commandant du pénitencier de Saint-Laurent, le 16 mai 1905, ANOM H 5204.

41. Liard-Courtois (2005, p. 223).

42. Jean-Marie Godefroy au commandant du pénitencier de Saint-Laurent, le 17 février 1903, ANOM H 5204

43. Bard, Chauvaud, Perrot, Petit (2002, p. 13).

44. Le ministre des Colonies au garde des Sceaux, le 16 octobre 1905, Archives Nationales (désormais AN) BB 182292. 
45. Le garde des Sceaux au président du Conseil, le 30 août 1906, AN BB 182292.

46. La commission de classement des récidivistes, composée d'experts désignés par le gouvernement, se réunit peu avant le départ d'un convoi et procède au classement à la relégation individuelle ou collective des relégués.

47. Mimande (1905, p. 753).

48. Le gouverneur au ministre des Colonies, le 8 avril 1914, ANOM H 5197.

\section{RÉSUMÉS}

La relégation des femmes récidivistes en Guyane demeure un épisode particulièrement tragique de l'histoire de la colonisation pénale française. 519 femmes ont été envoyées dans cette colonie à partir de 1887 pour être unies à des forçats et favoriser leur implantation durable pour constituer une colonie de peuplement. Mais internées au sein d'un dépôt couvent géré par des sœurs de l'œuvre de Saint-Joseph de Cluny, elles subissent dans les faits une peine de travail forcé d'une extrême sévérité. Et celles qui parviennent à échapper à la discipline du dépôt connaissent à l'extérieur des situations de misère sociale ou bien se retrouvent brutalisées ou prostituées par leurs maris. Cette expérience s'avère donc un échec complet et le gouvernement décide en 1905 d'abolir la relégation pour les femmes récidivistes.

The transportation of female recidivist criminals to French Guyana remains a particularly tragic chapter in the history of French penal colonies. From 1887 a total of 519 women were sent to this colony to join male convicts in an attempt to develop a permanent settlement. Yet, these women were interned within a closed institution run by nuns from the order of St. Joseph of cluny, where they were effectively subjected to extremely rigorous forced labour. Those who managed to escape the discipline within the institution, experienced social deprivation outside or found themselves brutalised by their husbands or forced into prostitution. As this scheme turned out to be a complete failure, the French government decided in 1905 to abandon further transportation of female recidivists.

\section{AUTEUR}

\section{JEAN-LUCIEN SANCHEZ}

Auteur d'une thèse d'histoire contemporaine intitulée La relégation des récidivistes en Guyane française. Les relégués au bagne colonial de Saint-Jean-du-Maroni, 1887-1953, Jean-Lucien Sanchez est membre de l'Institut de recherche interdisciplinaire sur les enjeux sociaux et actuellement enseignant vacataire en histoire contemporaine à Paris III Sorbonne-Nouvelle. Principales publications : L'anthropométrie au service de l'identification des récidivistes : l'exemple des relégués en Guyane française, in Piazza P. (dir.), Aux origines de la police scientifique : Alphonse Bertillon, précurseur de la science du crime, Paris, Karthala, 2011, pp. 189-201; La discipline au bagne colonial. Les relégués collectifs au pénitencier de Saint-Jean du Maroni, Guyane française, Revue Pénitentiaire, 2011, 10, pp. 67-87.

Jean-Lucien Sanchez 88 rue Myrha 
75018 Paris

jean_lucien_sanchez@yahoo.fr 